\title{
Desarrollo de la autonomía del niño en la atención pediátrica: hacia un modelo ético
}

Developing child autonomy in pediatric healthcare: towards an ethical model

\author{
Dr. Kyriakos Martakis, ${ }^{a, b, c}$ Dr. Helmut Brand ${ }^{a}$ y Dr. Peter Schröder-Bäck ${ }^{a, d}$
}

a. Departamento de Salud Internacional, Escuela del Instituto de Investigación de Atención y Salud Pública (Care and Public Health Research, CAPHRI), Maastricht. University, Holanda

b. Hospital de niños y adolescentes, Hospital Universitario de Colonia, Colonia.

c. Centro de prevención y rehabilitación, Hospital Universitario de Colonia, Colonia.

d. Facultad de Ciencias Humanas y de la Salud, Universidad de Bremen.

Alemania.

Correspondencia:

Dr. Kyriakos Martakis: kyriakos.martakis@ maastrichtuniversity.nl

Financiamiento:

Ninguno.

Conflicto de intereses: Ninguno que declarar.

Recibido: 18-7-2017

Aceptado: 8-11-2017

\section{RESUMEN}

Los cambios del nuevo Código Civily Comercial de la Argentina impulsa el desarrollo de la autonomía en niños y adolescentes. En este trabajo, estructuramos un modelo que describe la autonomía en la atención de la salud infantil. Hicimos un análisis bibliográfico enfocado en I) concepto de autonomía relacionado con el valor absoluto del individuo autónomo y II) el proceso de desarrollo de toma idónea de decisiones en función de la edad.

Resumimos nuestros hallazgos mediante el desarrollo de un modelo conceptual en el niño, el pediatra y los padres. La relación pediatraniño se fundamenta en formas de orientación y cooperación con diversos niveles de actividad y pasividad. La autoridad de los padres influye sobre el grado de autonomía en función del nivel de respeto e igualdad moral del niño.

$\mathrm{Al}$ aplicar el modelo para facilitar el diálogo entre pediatras, niños, padres y otros, se considerarán las circunstancias contextuales, existenciales, conceptuales y socio-éticas.

Palabras clave: adolescente, niño, autonomía personal, pediatría, proveedor de atención médica.

http:/ / dx.doi.org/10.5546/ aap.2018.e401

Texto completo en inglés:

http:/ / dx.doi.org/10.5546/ aap.2018.eng.e401

Cómo citar: Martakis K, Brand H, Schröder-Bäck P. Desarrollo de la autonomía del niño en la atención pediátrica: hacia un modelo ético. Arch Argent Pediatr 2018;116(3):e401-e408.

\section{INTRODUCCIÓN}

Según el Subcomité de Ética Clínica de la Sociedad Argentina de Pediatría (SAP), los cambios introducidos por el nuevo Código Civil y Comercial de la Nación afectan de manera importante la rutina pediátrica. ${ }^{1}$ Se considera que tanto niños como adolescentes son competentes en lo que respecta a las decisiones sobre su salud a partir de los trece años de edad, entretanto, su consentimiento y su asentimiento informados van adquiriendo mayor significado en la práctica pediátrica.
Junto con otras sociedades pediátricas nacionales, la SAP ha destacado la tarea pediátrica de impulsar la autonomía en los niños. En este documento, nos hemos enfocado en el concepto de "respeto por la autonomía" y los desafíos relevantes en la atención pediátrica.

En primer lugar, se objeta la edad a partir de la cual puede considerarse que la autonomía está suficientemente desarrollada para que se respeten las decisiones de los niños. En segundo lugar, el dipolo clásico (médico-paciente) se reemplaza por un triángulo de actores (niño/ adolescente, pediatra, padres). ${ }^{5} \mathrm{La}$ situación es similar a la que se da al objetar la autonomía en adultos menos autónomos, representados por tutores. ${ }^{6}$ Sin embargo, en nuestro caso, la autonomía aún está desarrollándose.

Por lo tanto, el objetivo fue presentar un modelo que describiera el desarrollo de la autonomía en la atención médica pediátrica. La idea es que sea una herramienta útil para entender las implicancias normativas de la autonomía infantil y para facilitar el debate.

\section{MÉTODOS}

Llevamos a cabo una investigación bibliográfica y un análisis de contenido de dirección. ${ }^{7}$ Examinamos los conceptos y los modelos que habían tenido gran influencia en la concepción de la autonomía, incluido el tema del paternalismo, definido como la desacreditación de la autonomía en pos de un supuesto beneficio para el individuo. Documentamos información 
pertinente en un corpus y estudiamos los datos utilizando métodos digitales de análisis cualitativo, siguiendo tres temas de estudio: las relaciones pediatra-niño, padre-niño y pediatrapadres. Limitamos nuestro estudio a situaciones que incluyeran a estos actores interactuando en un contexto justo, en el que se respetara la justicia legal, de derechos humanos y distributiva, ${ }^{8}$ así como el bien de la salud pública. ${ }^{9}$

Más aún, el modelo se desarrolló sintetizando los hallazgos clave (Figura 1). El modelo se sometió a varias fases de perfeccionamiento, incluida una presentación en la reunión de la Red de la Fundación Alemana para la Investigación Científica (Deutsche Forschungsgemeinschaft, DFG): "Children's well-being and child-protection: An analysis of the normative principles of children's wellbeing" (Bienestar y protección infantil: análisis de los principios normativos del bienestar infantil), ${ }^{10}$ que tuvo lugar del 4 al 6 de octubre de 2016, en Göttingen (Alemania).

\section{RESULTADOS}

A continuación, presentamos los hallazgos que consideramos pertinentes a la pregunta del estudio, ordenados de acuerdo con cuatro temas: a. autonomía e integridad moral del niño; b. rol de los padres; c. rol del pediatra; $y \mathrm{~d}$. beneficencia y el bien médico.

\section{¿Qué tan completo es completo?}

El desarrollo de la autonomía incluye niveles cada vez mayores de autodeterminación. Las decisiones cabales, la confianza en sí mismo y el equilibrio entre la presión de los padres y la de los pares son elementos clave en este proceso. ${ }^{11}$ Varios autores sostienen que aún no se han alcanzado la autonomía y la completitud para el paciente pediátrico. Esta posición puede explicarse por las teorías radicales que niegan el valor moral de los niños ${ }^{12-14} \mathrm{O}$ a través de la teoría de autonomía mínima, que atribuye una importancia absoluta a la edad cronológica. ${ }^{15}$ El carácter determinado por

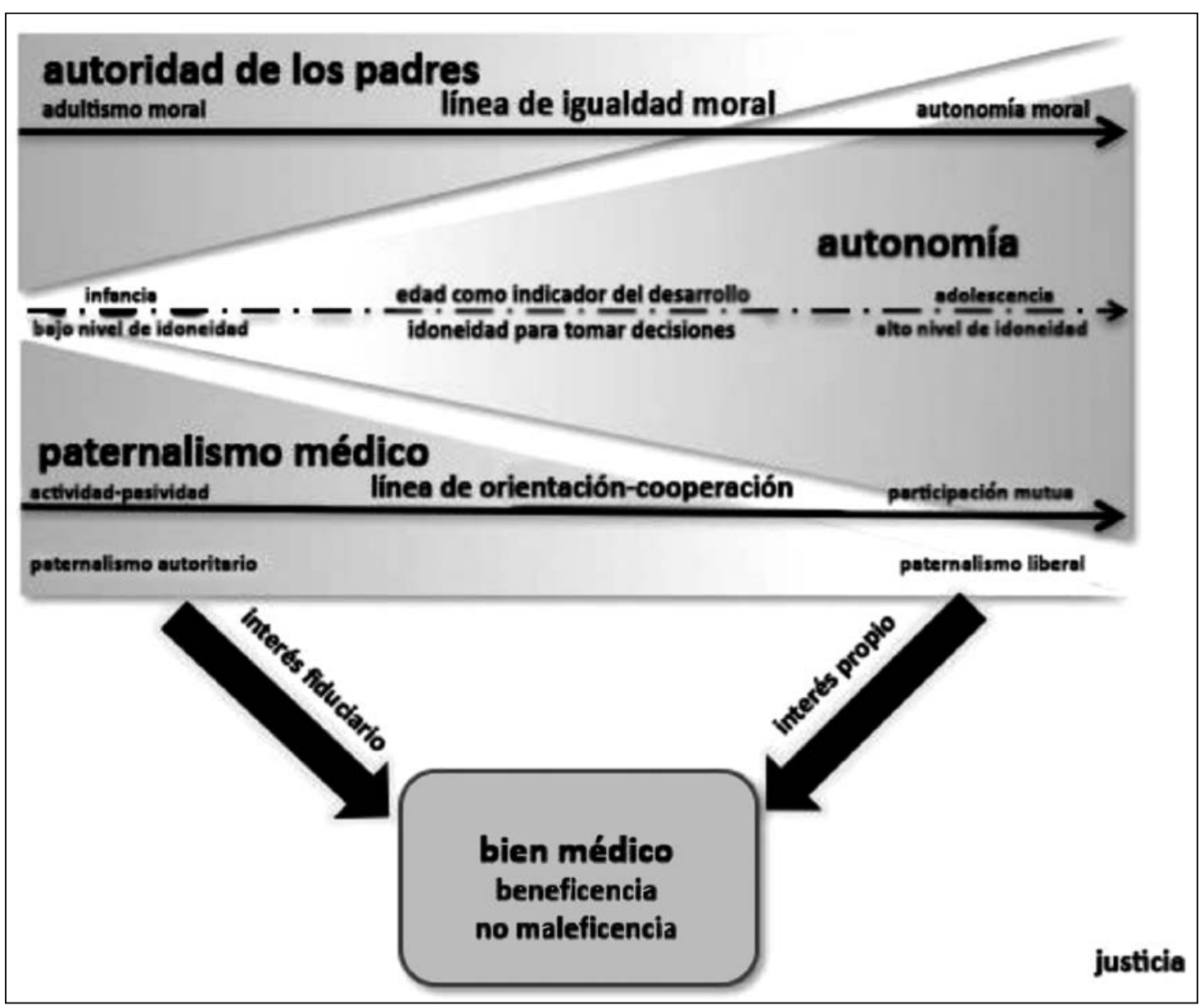


la edad quizá se asemeje a la controvertida teoría de Piaget sobre el desarrollo cognitivo. ${ }^{16}$ Resulta interesante observar que estudios recientes sobre la capacidad de los niños para tomar decisiones sugieren que las personas deben considerarse competentes para otorgar el consentimiento informado a partir de los 12 años de edad. ${ }^{17}$ Por lo tanto, la inteligencia podría complementar a la edad como factores clave en la idoneidad de los niños para consentir a la investigación. ${ }^{18}$

Por otro lado, el principio de "igualdad moral" representa un enfoque revolucionario. De hecho, el principio de "respeto por la autonomía" no solo equipara el valor moral de los niños de todas las edades y de los padres, sino también de los humanos de todas las edades. El papel que desempeñan los padres queda establecido al nacer el niño, a lo que sigue la confianza del niño, que es protegida por el respeto y el amor de los padres, valores que llevan a estos últimos a satisfacer las necesidades del niño. La negación del valor moral del niño, ya sea no intencional (negligencia) o intencional (abuso), es señal de traición. Por el contrario, solo podrá criarse un niño que actúe con resolución si los padres respetan la autonomía moral del niño. ${ }^{19}$

\section{La autoridad ¿define a los padres?}

La autoridad de los padres y los intereses familiares influyen sobre la autonomía del niño. La sobreprotección, por ejemplo, suele debilitar la capacidad del niño para desarrollar su autonomía, y los efectos difieren en función de la edad del niño y del nivel de sus capacidades físicas y cognitivas. ${ }^{20} \mathrm{Si}$ se tiene en cuenta la importancia de la edad y la inteligencia en el desarrollo cognitivo de los niños, los estilos sobreprotectores de crianza solo pueden ser contraproducentes. ${ }^{18}$ Por el contrario, las discusiones francas en el ámbito familiar y la participación en grupos centrados en la familia (que en ocasiones, incluso, llevan al niño a cuestionar las elecciones de sus padres) pueden resultar un medio importante para el desarrollo de la autonomía. ${ }^{21}$

Los estilos de crianza varían desde el autoritarismo absoluto hasta el gerenciamiento ${ }^{22}$ y los conceptos liberales. ${ }^{19}$ Los primeros, que incluyen expresiones como "adultismo moral", hasta pueden sostener que los niños son propiedad de los padres y que los padres pueden, por ejemplo, decidir si sus bebés deberían o no seguir viviendo. ${ }^{12} \mathrm{Sin}$ embargo, los conceptos modernos de crianza liberal se fundamentan en el principio de la confianza y el respeto por el valor moral del niño, en un plano de igualdad. ${ }^{19}$

\section{La función del pediatra}

La dinámica relación pediatra-niño se basa sobre la orientación y la cooperación. Así, el pediatra puede mostrar un nivel mayor o menor de autoridad, que resultará en formas más fuertes o más débiles de paternalismo médico, desde lo autoritario y lo coercitivo hasta lo limitante y lo liberal. ${ }^{23}$

De acuerdo con los modelos paternalistas tradicionales, el médico actúa en pos del bien del paciente (beneficencia) y está calificado para actuar en nombre del paciente e incluso para oponerse a la voluntad expresada por el paciente. Un paciente pasivo se limita a reconocer la autoridad del médico. ${ }^{24}$ No obstante, el cambio de paradigma que lleva a un comportamiento médico menos paternalista resulta en una variación en los niveles de actividad y pasividad por parte de ambos actores. En este contexto, el paternalismo puede asociarse directamente con el nivel de autonomía del niño. El paternalismo autoritario no contribuirá a desarrollar idoneidad para la toma de decisiones. En cambio, el paternalismo liberal es la fuerza que favorecerá en gran medida una participación pediatra-niño mutua en la toma de decisiones, y maximizará la capacidad del niño para ser autónomo. ${ }^{25}$ Pellegrino destaca que la relación pediatra-paciente se vincula con la idea de recuperar la completitud y la autonomía perdidas. ${ }^{26}$ De hecho, el pediatra no solo deberá favorecer la recuperación sino también estimular el desarrollo de mayor autonomía, en especial porque las enfermedades limitan existencialmente la autonomía de los niños.

Más aún, el asentimiento y el consentimiento informados son herramientas importantes que derivan del paternalismo liberal y facilitan de manera pedagógica el rol resolutivo del niño. Este rol se basa sobre el hecho de que los niños tienen sentimientos morales independientemente de la edad que tengan y que pueden actuar en consecuencia de formas que los demás pueden entender y compartir. ${ }^{19}$ De este modo, el pediatra tiene la obligación de explicarles utilizando términos apropiados para la edad y de solicitar el asentimiento de niños y adolescentes competentes. ${ }^{29}$

Además, los pediatras coordinan la interacción entre padres e hijos. ${ }^{30}$ Concentrándose en el niño dentro del seno del hogar, los pediatras facilitan el trabajo interdisciplinario necesario para contribuir a una crianza que fortalezca la autonomía. ${ }^{31}$ Esto no generará un aumento compensatorio de paternalismo médico. Además de las obligaciones con el "adulto emergente", los pediatras tienen obligaciones para con los padres, y eso incluye 
la disposición para debatir, consentir e, incluso, negociar responsabilidades entre los miembros de la familia o el equipo de proveedores de atención y los padres. Así, es necesario encontrar un equilibrio cuando se yuxtaponen los derechos que emanan de la autoridad paterna, los derechos de los nuevos padres y la autonomía del niño. ${ }^{33}$

Por último, la función del pediatra es fundamental en los casos de irreconciabilidad absoluta entre los padres y los niños. De hecho, es determinante tener el derecho a solicitar la intervención del organismo local para el bienestar de los niños y los jóvenes, a consultar a un comité de ética, o, incluso, a iniciar una intervención legal. ${ }^{1}$ No obstante, los pediatras no siempre reciben el apoyo social y legal adecuado que les permita utilizar dichas herramientas. ${ }^{2}$

\section{Bien médico}

Se objeta que la beneficencia y la no maleficencia ${ }^{26}$ son elementos generadores de paternalismo. En el contexto de la atención de la salud, el niño, el médico y los padres interactúan libremente por el bien médico del niño. ${ }^{19}$ De este modo, todos los actores tienen como objetivo maximizar los beneficios netos y minimizar los daños netos derivados de distintas causas. La fuerza que impulsa al pediatra y a los padres es el interés fiduciario, mientras que la que motiva al niño es el propio interés. ${ }^{4}$

\section{Esbozo del modelo}

La terminología que describe el desarrollo de la autonomía en la bibliografía es variable y refleja diferencias en la postura de los académicos. De hecho, los términos "autonomía futura" y "autonomía en desarrollo" a menudo se usan en forma intercambiable. En nuestra opinión, esto no es del todo correcto. La "autonomía futura" es estática, ilusoria, e ignora al niño dado que se refiere solo al adulto que ese niño será. Derivada del adultismo moral, no representa el tipo de autonomía al que debería darse prioridad. "Autonomía en desarrollo", en cambio, se refiere a un proceso dinámico que conduce a la autonomía a través de la confianza y el cuidado, la orientación y la cooperación. La meta no debería ser solo la preparación para una adultez autónoma, sino la salvaguarda de la autonomía a cada paso: desde la infancia hasta la adultez.

Además, en la bibliografía se describen dos ideas principales y de algún modo contradictorias relacionadas con la autonomía. En la primera, y como expresión de la autonomía kantiana, el concepto de autonomía se refiere al valor absoluto del individuo autónomo. ${ }^{19}$ La segunda refleja el proceso de desarrollo de la toma competente de decisiones como una función de la edad, tal como la presentan Beauchamp y Childress. ${ }^{3}$

Entonces, ¿es la edad cronológica tan importante para el desarrollo de la autonomía? En nuestra opinión, la edad de desarrollo del niño, que resume los factores de edad y desarrollo cognitivo, podría ser la clave para evaluar el desarrollo de funciones asociadas con la autonomía, tales como la idoneidad para tomar decisiones. Es por este motivo que hemos incluido ambas ideas en el modelo (Figura 1) usando tanto el eje de igualdad moral (enunciando que todos los niños tienen el mismo valor moral pese a las diferencias en sus capacidades), como el eje de capacidad para tomar decisiones como función de la "edad de desarrollo". En el futuro, la investigación tendrá en cuenta la edad de desarrollo del niño en lugar de la edad cronológica, y utilizará las herramientas de evaluación apropiadas.

En un intento por exponer gráficamente distintos niveles de paternalismo frente al grado de autonomía, y utilizando una línea de orientación-cooperación como eje principal, nos dimos cuenta de que el paternalismo liberal será el paradigma para promover activamente la autonomía en desarrollo de los niños. Los pediatras cooperarán con los padres para empoderar a los jóvenes pacientes en su camino hacia la autonomía.

$\mathrm{Al}$ aplicar el modelo, es necesario abordar una serie de circunstancias. Pellegrino las clasificó como contextuales, existenciales, conceptuales y socio-éticas. ${ }^{26}$ En la Tabla 1 se resumen los temas relevantes que surgen y deben considerarse.

\section{Implementación}

En los dos casos siguientes, que enuncian diferentes aspectos, hemos ejemplificado desafíos relacionados con la autonomía y los discutimos en las distintas circunstancias construidas, tal como lo describe el modelo.

\section{Caso 1: Vacuna contra la gripe}

Verónica es una niña de diez años. Su mejor amiga, Mariella, una paciente con atrofia muscular espinal ( $A M E$ ) tipo III, suele tener infecciones graves de las vías respiratorias bajas. Verónica siempre visita a Mariella cuando está hospitalizada. Verónica desea recibir la vacuna intramuscular contra la gripe estacional porque está convencida de que aumentar la inmunidad colectiva es fundamental para pacientes 
como Mariella. Sus padres son escépticos respecto de la vacunación.

Circunstancias contextuales: El pediatra primero deberá abordar los temas de variabilidad del contexto. La enfermedad puede, en sí misma, ser importante para Verónica. No obstante, las consecuencias en niños sanos son limitadas. Los efectos secundarios de la vacuna son inocuos: incluyen infecciones leves de las vías respiratorias y fiebre, y lo más probable es que no representen una amenaza para su autonomía en desarrollo. No se ha probado que las vacunas intranasales sean eficaces. Brindar información basada en la evidencia descartará problemas relacionados con la falta de conocimiento sobre salud. La idoneidad de la niña y de los padres para tomar decisiones puede examinarse con herramientas estandarizadas. La bibliografía sugiere que un niño de diez años de edad no siempre está capacitado para razonar sabiamente en este contexto.
Circunstancias existenciales: Por el otro lado, la exposición que Verónica ha tenido a los peligros de la gripe entre los pacientes con AME ha aumentado su conciencia respecto del tema. En su conversación con Verónica, el pediatra desechó las dudas respecto de su idoneidad para tomar decisiones. Aparentemente, la edad cronológica no es la dimensión más importante.

Verónica es una persona sana, de modo que no surgiría ningún problema importante relacionado con la vacunación. Según las recomendaciones nacionales, la vacuna debería aplicarse cada año a todos los niños desde los dos hasta los seis años de vida.

Circunstancias conceptuales: El caso aborda, fundamentalmente, el cambio de paradigma de la cura a la atención. La vacunación puede, sin duda, aumentar la inmunidad colectiva y el bienestar de Verónica no corre ningún riesgo.

Por otro lado, los padres de Verónica expresan preocupación respecto de su ausentismo escolar. Según

TABLA 1. Ejemplos de circunstancias que deben abordarse al aplicar el modelo

Circunstancias contextuales

Circunstancias existenciales

Circunstancias conceptuales

Circunstancias socio-éticas
- Variabilidad del contexto

- Naturaleza de la enfermedad, probabilidades de cura, urgencia, prognosis y consecuencias para la autonomía en desarrollo del niño.

- Propiedades de los diagnósticos o los tratamiento ofrecidos y consecuencias para la autonomía en desarrollo del niño.

- Tratamientos o métodos de diagnóstico alternativos que se ofrecerán y consecuencias para la autonomía en desarrollo del niño.

- Nivel de conocimientos sobre salud que tienen el niño y los padres.

- Capacidades del niño y los padres para tomar decisiones.

- Considerar el inconveniente de las falsas generalizaciones.

- De qué manera la enfermedad en sí misma puede cambiar las ideas y la idoneidad para tomar decisiones tanto del niño como de los padres.

- De qué manera el tratamiento y los tratamientos alternativos pueden cambiar las ideas y la idoneidad para tomar decisiones tanto del niño como de los padres.

- Efecto del conocimiento previo o de la exposición previa a la enfermedad o el tratamiento sobre el nivel de conocimientos de salud del niño y los padres.

- Considerar las diferencias institucionales o sociales que afectan la transferibilidad de un tratamiento o terapia en otro contexto.

- Cambio de paradigma: de la cura a la atención, y cómo se aplica en el caso.

- Respeto por la no maleficencia, si no por la beneficencia, del niño y la familia.

- Respeto por la medicina basada en la evidencia cuando se establecen indicaciones.

- ¿Existe el problema de que el bien del individuo o el bien médico estén viéndose perjudicados por el bien común?

- ¿Existe algún problema respecto del interés de los padres?

- ¿Existe algún problema respecto de los motivos del niño?

- ¿Existe algún problema respecto de la exposición de antecedentes familiares o de información sobre terceros?

- ¿Existe algún problema ético respecto de la financiación del tratamiento o del diagnóstico? ¿Quién se hace cargo de los costos?

- ¿Es un caso de absolutización de la autonomía?

- ¿Es un caso en el que se actúa sin respetar un marco de justicia (legal, de derechos humanos y distributiva)? 
parece, Verónica ha faltado a clase con frecuencia en las últimas semanas. En este momento, su asistencia es regular. A la madre le preocupa que Verónica pueda presentar fiebre y necesite quedarse en casa después de la vacunación. Es posible que Verónica no pueda terminar su curso si vuelve a surgir el tema del ausentismo en la escuela.

Circunstancias socio-éticas: El pediatra deberá abordar concienzudamente el tema del ausentismo escolar, que pone en riesgo la autonomía en desarrollo de Verónica. No hay problemas con respecto al interés de los padres. Sin embargo, las razones de Verónica deberán volver a evaluarse, posiblemente, conversando con ella a solas. Verónica lamenta faltar a clase, y afirma que la solidaridad es su única razón. Se sentiría satisfecha si recibiera la vacuna durante las vacaciones de invierno, de modo que no tuviera que faltar a la escuela a causa de una infección posterior a la vacunación.

Verónica desea pagar ella misma la vacuna, usando un dinero que ella y Mariella reunieron. Excepto por el hecho de que el pediatra ahora conoce la situación de Mariella, no hay ningún otro problema respecto de la exposición de antecedentes familiares o información sobre

TABLA 2. Estudio del caso vacuna contra el virus papiloma humano

Circunstancias contextuales

Circunstancias existenciales

Circunstancias conceptuales

Circunstancias socio-éticas
- Los tipos de cáncer causados por el VPH presentan un riesgo grave de una enfermedad que con frecuencia no puede curarse correctamente, y que puede prevenirse con eficacia mediante la vacuna contra el VPH.

- En Argentina, la cobertura de la vacuna es alta. Se espera lograr un aumento significativo de la inmunidad colectiva en las próximas décadas.

- La prevención de la transmisión vaginal, por ejemplo, a través del uso de preservativo, con frecuencia no previene las manifestaciones orofaríngeas.

- La inactividad sexual o la abstinencia son presentadas como alternativas por la niña misma. Sin embargo, esta alternativa es, sin duda, una restricción para su desarrollo futuro, tanto personal como social, y también para su autonomía en desarrollo.

- En este caso, el problema parece ser el temor nacido del desconocimiento de temas de salud.

- En casos como este, la charla privada con el pediatra es una herramienta muy importante, que debe ser utilizada.

- No hay ninguna enfermedad subyacente ni experiencia previa que pudiera influir en la idoneidad de la niña para tomar decisiones ni en su nivel de autonomía.

- Wenzeslava no estuvo expuesta anteriormente a enfermedades causadas por el VPH. Sin embargo, sí estuvo expuesta a información falsa sobre los posibles efectos secundarios de la vacuna.

- Existen diferencias sociales obvias entre los dos contextos. La vacuna contra el VPH forma parte de un programa cuya implementación ha sido un éxito en Argentina, pero no ha sido así en muchos otros países. Es necesario tratar minuciosamente los problemas de comunicación o la falta de confianza asociados con la naturaleza intercultural de la interacción pediatra-paciente.

- El respeto por la no maleficencia de la niña es fundamental. Wenzeslava debe tener garantías de que los efectos secundarios que teme no están asociados a la vacuna contra el VPH. Sin embargo, se le dará información sobre todos los efectos secundarios que sí son reales, incluidos aquellos que sean extremadamente infrecuentes.

- La prevención de los tipos de cáncer generados por el VPH refleja el respeto por la beneficencia y también por la medicina basada en la evidencia.

- Cambio evidente de paradigma: de cura a atención. Wenzeslava no recibirá la vacuna con la intención de administrar el tratamiento de inmediato. Ella entenderá cuáles son los beneficios personales y sociales de esta decisión, y podrá recibir la vacuna más adelante.

- La negociación con la niña sería una herramienta útil.

- Que el bien del individuo o el bien médico estén viéndose perjudicados por el bien común no es motivo de preocupación.

- Ni el interés de los padres ni los motivos de la niña son motivo de preocupación.

- No hay problema respecto de la exposición de antecedentes familiares o de información sobre terceros.

- La financiación del tratamiento o del diagnóstico no es motivo de preocupación.

- No es un caso de absolutización de la autonomía, y el marco de justicia se ha respetado en todo momento. 
terceros. El marco de justicia se respetó en todo momento.

Por lo tanto, podríamos llegar a la conclusión de que la participación del pediatra y del niño en el marco de la relación de orientación-cooperación condujo a un compromiso que impulsa la autonomía de Verónica, dado que su asistencia a clases no se puso en peligro y que ella misma contribuye económicamente a su tratamiento. Los motivos de preocupación de los padres se consideraron con seriedad y se resolvieron eficazmente. Su decisión final refleja la aceptación de la capacidad de resolución de Verónica.

\section{Caso 2: Vacuna contra el virus papiloma humano}

El virus papiloma humano (VPH) suele ser causa de cáncer en las mujeres en edad de procrear. Desde 2011, la vacuna contra el VPH se ofrece como parte del programa nacional de vacunación en Argentina, y la tasa de cobertura es alta. ${ }^{34}{ }^{34}$ Wenzeslava es una joven de 15 años, inmigrante búlgara. Su familia se mudó a la Argentina hace un par de meses. Sus padres quieren que Wenzeslava se vacune. Sin embargo, ella se niega, y su argumento es que aún no está sexualmente activa. En una conversación privada con el pediatra, sin embargo, admite que tiene temor por los posibles efectos secundarios. Wenzeslava oyó que, en Bulgaria, una niña desarrolló granulomatosis de Wegener después de vacunarse contra el VPH. Si bien no habia una relación causal entre ambos eventos, las críticas de los medios de comunicación búlgaros sobre la vacuna han fortalecido el alto escepticismo ${ }^{35} 35$ que ya existía.

En la Tabla 2 se resumen las circunstancias que debieron abordarse.

En este caso, identificamos problemas de desconocimiento de la salud, temor inducido por los medios de comunicación que se oponen a una vacuna contra un agente de transmisión sexual, problemas respecto de la interacción intercultural, barreras idiomáticas y probable falta de confianza en su nuevo pediatra. El pediatra deberá abordar todos estos aspectos de manera minuciosa. Los padres entienden la indicación y están a favor del tratamiento. Por el otro lado, no puede ignorarse el argumento esgrimido por Wenzeslava respecto de que no está sexualmente activa. La niña está en una edad en que se la considera idónea para tomar decisiones como esa (y seguramente lo es).

La negociación parece ser la salida. El pediatra informará cuáles son los beneficios y los riesgos de la vacuna, y programará una cita para volver a evaluar la situación, por ejemplo, seis meses después. Deberá dejarse en claro que es absolutamente recomendable que se vacune cuando planee estar sexualmente activa. Se debe informar a los padres que, llegado el caso, la vacuna puede aplicarse incluso sin que ellos participen en la decisión.

\section{CONCLUSIONES}

Creamos un modelo para explorar los problemas que surgen con el desarrollo de la autonomía y la tensión con el paternalismo, impulsado tanto por la autoridad del pediatra como la de los padres. Este modelo deberá servir de base para el diálogo interdisciplinario entre los actores involucrados, como pediatras, médicos de salud pública, especialistas en ética, padres e, incluso, los mismos niños. Al aplicar el modelo, deberán evaluarse con sentido crítico las circunstancias contextuales, existenciales, conceptuales y socio-éticas.

\section{Agradecimiento}

Agradecemos profundamente a quienes participaron en el congreso anual del proyecto "Children's well-being and child-protection: An analysis of the normative principles of children's well-being" (Bienestar y protección infantil: análisis de los principios normativos del bienestar infantil), llevado a cabo en Göttingen, del 4 al 6 de octubre de 2016, por su opinión sobre un borrador anterior de este modelo. Agradecemos su apoyo a la Dra. Kruthika Thangavelu, la Dra. Mahima Venkateswaran y la Dra. Verónica Maurino.

\section{REFERENCIAS}

1. Subcomisión de Ética Clínica. Sociedad Argentina de Pediatría. The principle of autonomy in pediatrics under the new Civil and Commercial Code of the Nation: a communication of the Subcommittee on Clinical Ethics. Arch Argent Pediatr 2016;114(5):485-8.

2. Gahr M. Patientenverfügungen von Minderjährigen. Monatsschr Kinderheilkd 2015;163(4):375-378.

3. Beauchamp TL, Childress JF. Principles of biomedical ethics. $7^{\text {th }}$ ed. Oxford: Oxford University Press; 2013.

4. O'Neill O. Autonomy and trust in bioethics. J $R$ Soc Med 2002;95(8):423-4

5. Carse AL, Nelson HL. Rehabilitating care. Kennedy Inst Ethics J 1996;6(1):19-35.

6. Elliott BA, Gessert CE, Peden-McAlpine C. Family decisionmaking in advanced dementia: narrative and ethics. Scand J Caring Sci 2009;23(2):251-8.

7. Hsieh HF, Shannon SE. Three approaches to qualitative content analysis. Qual Health Res 2005;15(9):1277-88.

8. Gillon R. Medical ethics: four principles plus attention to scope. BMJ 1994;309(6948):184-8.

9. O'Neill O. Public health or clinical ethics: thinking beyond borders. Ethics Int Aff 2002;16(2):35-45.

10. Bagattini A. DFG-Projekt: Kindeswohl und Kinderschutz - Analyse der normativen Grundlagen des Kindeswohls. 2017. [Acceso: 15 de noviembre de 2017] Disponible en: http: / / gepris.dfg.de/gepris / projekt/ 263376822. 
11. Russell ST, Bakken RJ. Development of autonomy in adolescence. Cooperative Extension, Institute of Agriculture and Natural Resources, University of Nebraska-Lincoln;2002. [Acceso: 15 de noviembre de 2017] Disponible en: http:/ / www.basicknowledge101.com/ pdf/Development $\% 20$ of $\% 20$ Autonomy $\% 20 \mathrm{in} \% 20$ Adolescence.pdf

12. Giubilini A, Minerva F. After-birth abortion: why should the baby live? J Med Ethics 2013:39(5):261-3.

13. Schapiro T. What Is a Child? Ethics 1999;109(4):715-38.

14. Tristram E. The foundations of bioethics. $2^{\text {nd }} \mathrm{ed}$. Oxford: Oxford University Press; 1996.

15. Jaworska A. Caring, minimal autonomy, and the limits of liberalism. In: Lindemann H, Verkerk M, Walker M eds. Naturalized Bioethics: Toward Responsible Knowing and Practice. Cambridge: Cambridge University Press, 2009: 80-105.

16. Piaget J. Piaget's theory. In: Mussen P (ed). Handbook of child psychology. $4^{\text {th }}$ ed. New York: Wiley; 1983.

17. Hein IM, De Vries MC, Troost PW, et al. Informed consent instead of assent is appropriate in children from the age of twelve: Policy implications of new findings on children's competence to consent to clinical research. BMCMed Ethics 2015;16(1):76.

18. Hein IM, Troost PW, Lindeboom R, et al. Key factors in children's competence to consent to clinical research. BMC Med Ethics 2015;16(1):74.

19. Wiesemann C. Moral Equality, Bioethics and the Child. Switzerland: Springer; 2016.

20. Affdal AO, Moutard ML, Billette de Villemeur T, et al. Le difficile passageàl'âge adulteen matière de soins:l'exemple de l'épilepsie. Arch Pediatr 2015;22(4):337-42.

21. Nichol J, Thompson EA, Shaw A. Beliefs, decision-making, and dialogue about complementary and alternative medicine (CAM) within families using CAM: a qualitative study. J Altern Complement Med 2011;17(2):117-25.

22. Brennan S, Noggle R. The moral status of children: Children's rights, parents' rights, and family justice. Soc
Theor Pract 1997;23(1):1-26.

23. Strasser M. The new paternalism. Bioethics 1988;2(2):103-17.

24. Komrad MS. A defence of medical paternalism: maximising patients' autonomy. J Med Ethics 1983;9(1):38-44.

25. KarlssonK.Handling dilemmas of self-determination in'usercentred' rehabilitation. Disabil Rehabil 2007;29(3):245-53.

26. Pellegrino ED, Thomasma DC. The conflict between autonomy and beneficence in medical ethics: proposal for a resolution. J Contemp Health Law Policy 1987;3:23-46.

27. Kuther TL, Posada M. Children and adolescents' capacity to provide informed consent for participation in research. Adv Psychol Res 2004;32:163-73.

28. Rossi WC, Reynolds W, Nelson RM. Child assent and parental permission in pediatric research. Theor Med Bioeth 2003;24(2):131-48.

29. Sibley A, Pollard AJ, Fitzpatrick R, et al. Developing a new justification for assent. BMC Med Ethics 2016;17:2.

30. Simpson C. Supporting the Developing Autonomy of Youth Patients: Reflections on the Role of Healthcare Providers. In: Zlotnik Shaul R. (ed). Paediatric Patient and Family-Centred Care: Ethical and Legal Issues. New York: Springer; 2014. Págs.175-84.

31. Harrison C, Kenny NP, Sidarous M, et al. Bioethics for clinicians: 9. Involving children in medical decisions. CMAJ 1997; 156(6):825-8.

32. Kloep M, Hendry LB. Letting go or holding on? Parents' perceptions of their relationships with their children during emerging adulthood. Br J Dev Psychol 2010;28(Pt 4):817-34.

33. Goering S. Postnatal reproductive autonomy: promoting relational autonomy and self-trust in new parents. Bioethics 2009;23(1):9-19.

34. Patel H, Wilson E, Vizzotti C, et al. Argentina's Successful Implementation Of A National Human Papillomavirus Vaccination Program. Health Aff(Millwood) 2016;35(2):301-8.

35. Petrova D, Brunton CG, Jaeger M, et al. The Views of Young Women on HPV Vaccine Communication in Four European Countries. Curr HIV Res 2015;13(5):347-58 